\title{
Intense Visible Luminescence in CdSe Quantum Dots by Efficiency Surface Passivation with $\mathrm{H}_{2} \mathrm{O}$ Molecules
}

\author{
Hyeoung Woo Park and Do-Hyung Kim \\ School of Physics and Energy Science, Kyungpook National University, Daegu 702-701, Republic of Korea \\ Correspondence should be addressed to Do-Hyung Kim, kimdh@knu.ac.kr
}

Received 27 July 2011; Accepted 23 October 2011

Academic Editor: Laécio Santos Cavalcante

Copyright ( $) 2012$ H. W. Park and D.-H. Kim. This is an open access article distributed under the Creative Commons Attribution License, which permits unrestricted use, distribution, and reproduction in any medium, provided the original work is properly cited.

We have investigated the effect of water $\left(\mathrm{H}_{2} \mathrm{O}\right)$ cooling and heat treatment on the luminescence efficiency of core CdSe quantum dots (QDs). The photoluminescence (PL) quantum yield of the CdSe QDs was enhanced up to 85\%, and some periodic bright points were observed in wide color ranges during the heat treatment of QDs mixed with $\mathrm{H}_{2} \mathrm{O}$. The PL enhancement of QDs could be attributed to the recovery of QDs surface traps by unreacted ligands confined within the hydrophilic $\mathrm{H}_{2} \mathrm{O}$ molecule containers.

\section{Introduction}

Colloidal semiconductor nanocrystals, so-called quantum dots (QDs), have generated tremendous interest both for fundamental research and technical applications such as light emitting diodes (LEDs) [1], bioimaging [2], and solar cells [3]. Because of their size-dependent photoluminescence (PL) related to quantum-size effect tunable across the wide visible spectrum, CdSe QDs have become the most extensively investigated QDs [4]. Since core CdSe QDs usually have low luminescence efficiency, much experimental work has been devoted to surface passivation to improve the luminescence efficiency of QDs. Several methods of organic and inorganic surface modification have been developed to mediate the problems by passivation of the surface defects of QDs. The organic passivation methods such as polymer encapsulation, surface grafting, and ligand exchange [5-7] have usually accompanied a decrease in quantum yield (QY) and an undesirable size increase relative to the original QDs and more QDs being included in the polymer encapsulation. It is well know that shelling of inorganic atomic layers with a wider bandgap on the surface of core QDs effectively reduces nonradioactive recombination which results in the enhancement of PL QY $[8,9]$. The QY reported values are generally lower than $50 \%$ for nonpassivation QDs and the best reported values of QY are approximately $\sim 80 \%$ for inorganic-shelled QDs [9, 10]. For this reason, the effects and methods for shelling on the surface of core QDs have been intensively studied. However, additional inorganic shelling has to be delicately controlled to obtain the desirable atomic layers and can have possible problems such as lattice mismatch (limited materials), FWHM broadening, and size increases. Moreover, the various shelled QDs still have surface state problem such as PL decrease of multishell QDs at ligand exchange process [11]. QDs are commonly prepared by organometallic chemistry using a mixture of trioctylphosphine (TOP) and trioctylphosphine oxide (TOPO) as the prototypical ligand system [10]. These ligands as the capping group provide colloidal stability in organic solvents such as chloroform, hexane, and toluene and serve as agents for reducing the undesirable electronic effects related to dangling bonds or surface states. Although the surfaces of the QDs are passivated by these organic ligands during the synthesis process, the PL QY of the QDs is usually very low. There was a report about the "bright point" of core QDs, in which the QY reached approximately $\sim 80 \%$ by adjusting the chemical ratio, synthesis time, and temperature [12]. However, the wavelength showing the "bright point" was limited to orange color range at about $\sim 610 \mathrm{~nm}$. It could also be very effective to enhance the PL QY by reducing some or the entire surface trapping states of the low QY QDs through a simpler posttreatment instead of searching for complex 

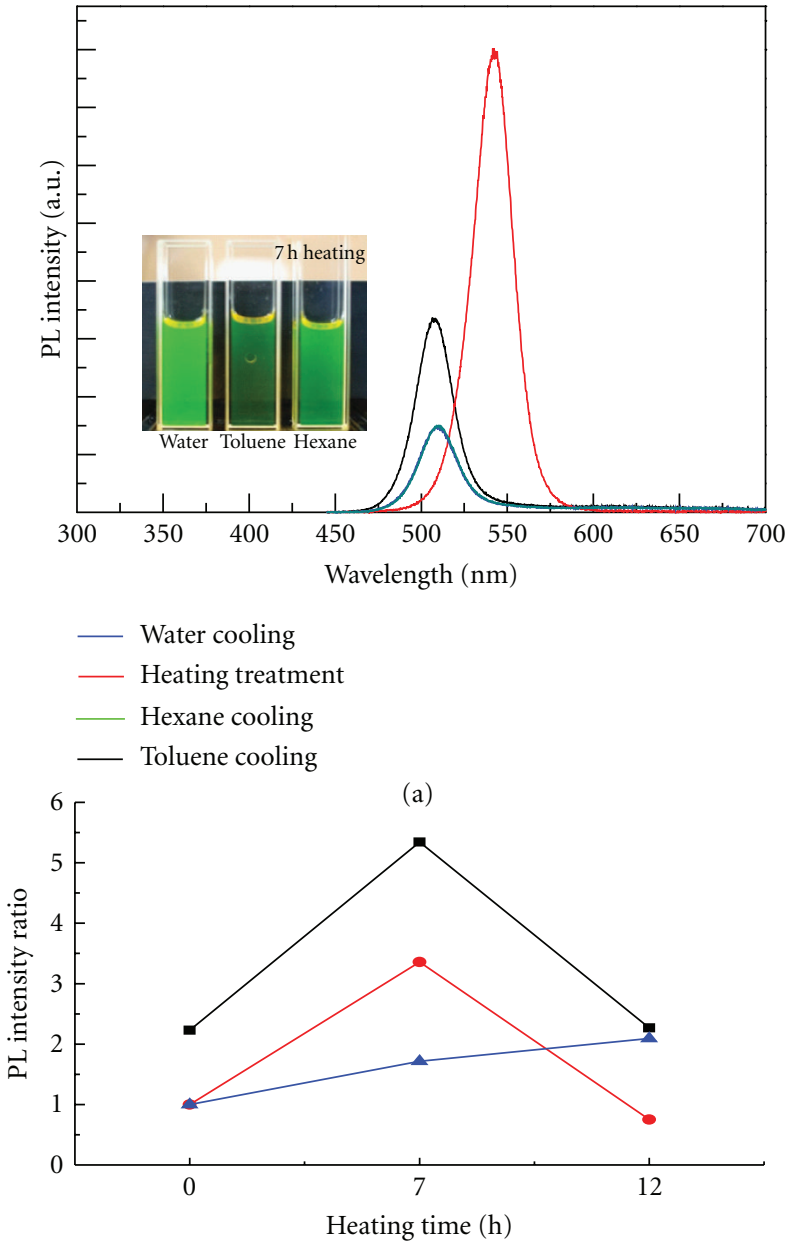

(c)
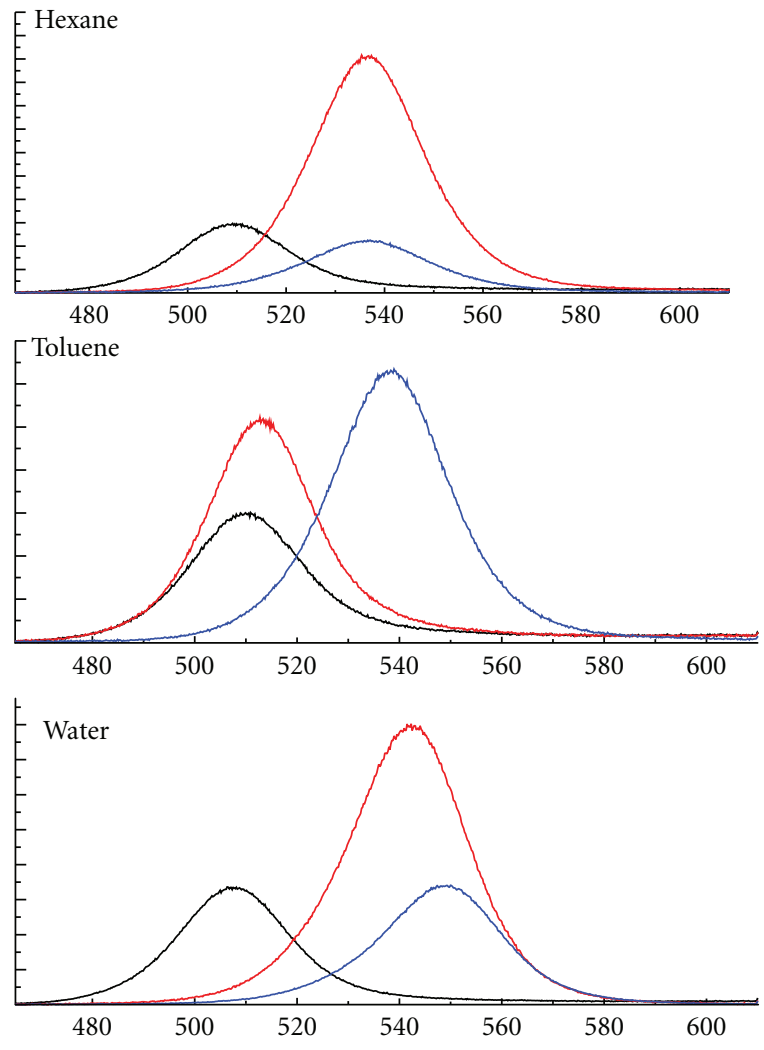

No heating
$-7 \mathrm{~h}$ heating
$-12 \mathrm{~h}$ heating

(b)

Figure 1: (a) The PL spectra of the QDs cooled by different solvents and additional heat treatment, (b) the change in PL spectra after additional heat treatment of the QDs in different solvents, (c) the ratio of PL peak intensity calculated from the experimental date of (b). The inset of (a) is the photograph of core CdSe QDs under room light.

synthesis conditions. Therefore, in this communication, we have designed $\mathrm{H}_{2} \mathrm{O}$ containers as a mechanism in order to recovery surface trap states effectively. We have improved dramatically the PL QY of the core CdSe QDs up to $~ 85 \%$ in the green color range which was not in the reported bright point widow of the orange color.

\section{Experimental Procedures}

A $2 \mathrm{M}$ stock solution of trioctylphosphine selenide (TOP : Se) was prepared by dissolving $15.8 \mathrm{~g}$ of Se into $100 \mathrm{~mL}$ of TOP. The stock solution was filled in $100 \mathrm{~mL}$ three-neck roundbottomed flask and fitted with a thermocouple temperature sensor and condenser, with TOPO (10 g), HDA (10 g) and TOP $(2.5 \mathrm{~mL})$, and heat to $170^{\circ} \mathrm{C}$ under vacuum for $1-2 \mathrm{~h}$ and raise the temperature to $340-350^{\circ} \mathrm{C}$. In a separate vial, mix Cd(acac $)_{2}(620 \mathrm{mg}), \mathrm{HDDO}(1 \mathrm{~g})$, and TOP $(5 \mathrm{~mL})$ and heat under vacuum to $100^{\circ} \mathrm{C}$; the solution should become homogeneous. Cool the mixture to approximately $80^{\circ} \mathrm{C}$ and add $5 \mathrm{~mL}$ of a $2 \mathrm{M}$ TOP: Se. The solution mixture of cadmium and selenium precursors was rapidly injected into the hot flask containing the coordinating solvent, and then cooled using each solvents. The QDs extracted from same hot batch were divided into different cooling solvents. The QDs with different cooling solvents were heated in vacuum oven under air condition at $120^{\circ} \mathrm{C}$.

\section{Results and Discussion}

Figures 1(a) and 1(c) show a 2-fold increase in the PL intensity obtained by changing the cooling process. The 5fold enhancement in the PL intensity was finally achieved by additional heat treatment. Diverse solvents such as toluene, hexane, methanol, ethanol, acetone, and $\mathrm{H}_{2} \mathrm{O}$ were applied to confirm the effect of the cooling process on the enhancement of the PL intensity of the QDs (Figure 2(a)). Toluene and hexane were most frequently used in the cooling process because the QDs dissolved very well in these solvents. 


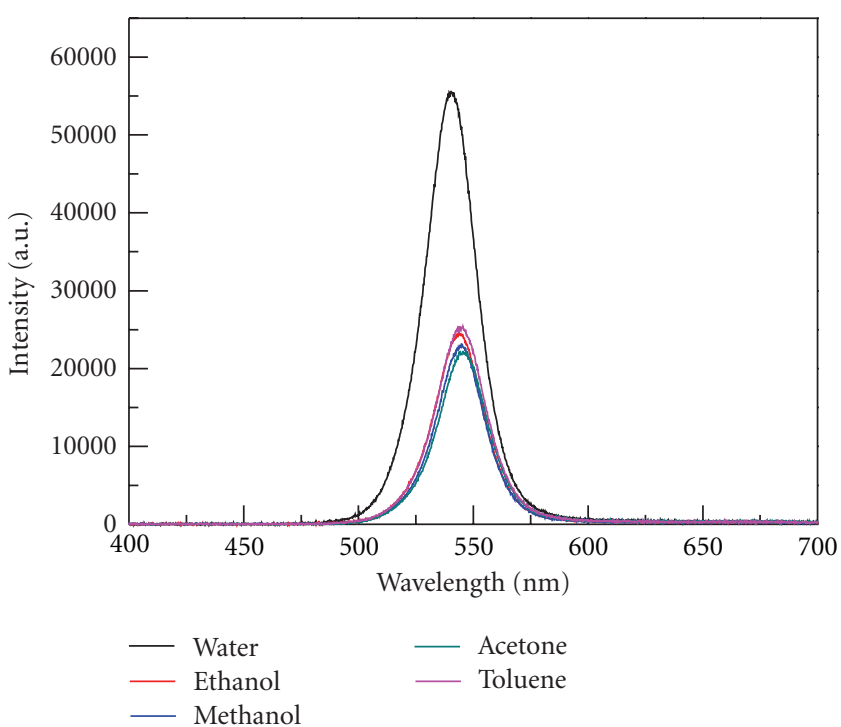

(a)

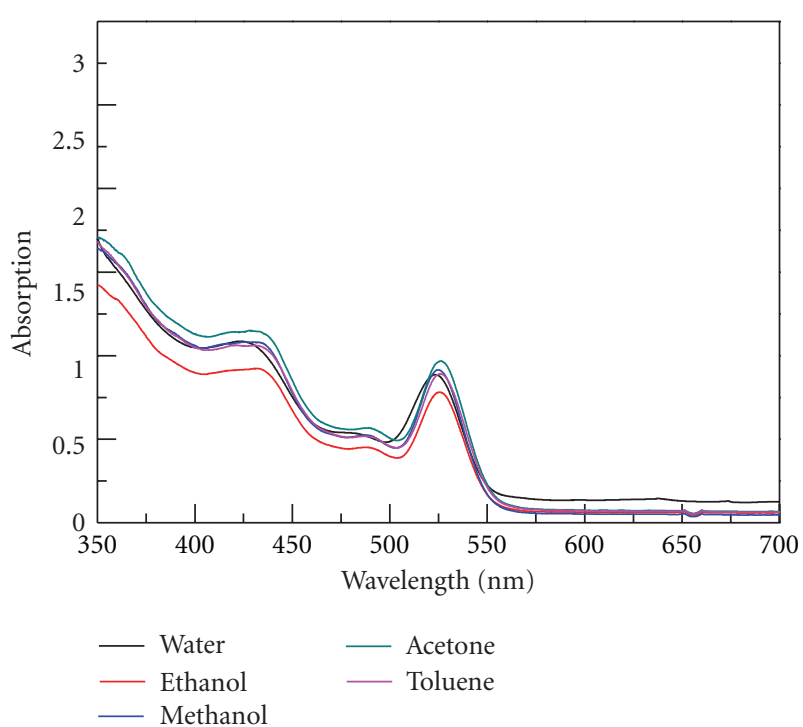

(b)

Figure 2: (a) Absorption and (b) PL spectra of QDs cooled by diverse solvents (toluene, hexane, methanol, ethanol, acetone, and $\mathrm{H}_{2} \mathrm{O}$ ).

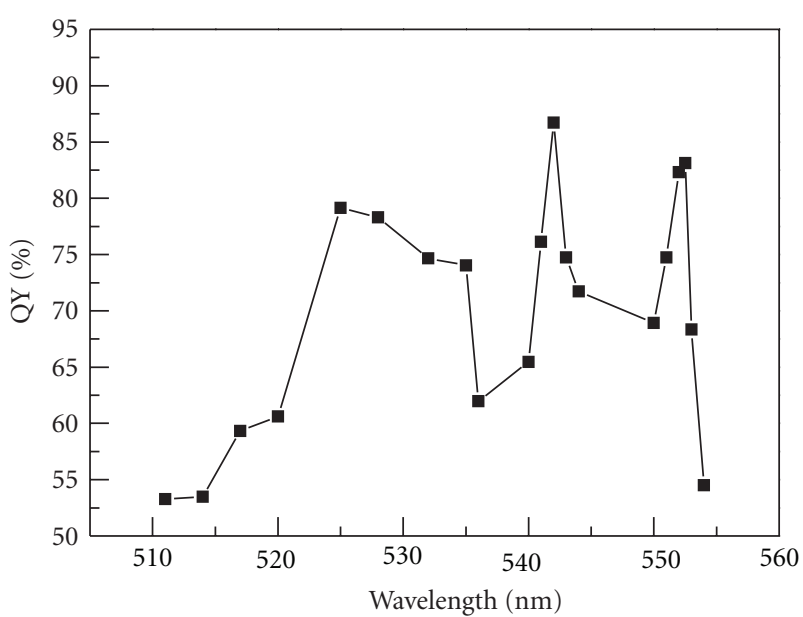

FIGURE 3: The QY and red-shift of CdSe QDs during heat treatment. QYs calculated by comparison of the standard dye Rhodamine 6G.

It was also confirmed that the extracting orders of the QDs and transferring orders to the solvents did not have a significant effect on the PL properties of the QDs. Therefore, the cooling process was the main parameter for comparing the PL properties of the QDs in these experiments. The supernatant QDs collected after mixing vigorously with $\mathrm{H}_{2} \mathrm{O}$ were redispersed in toluene or hexane to measure the PL. The concentration of the QD samples was adjusted by the absorption peak of the QDs previously described elsewhere (Figure 2(b)) [13]. The PL enhancement in $\mathrm{H}_{2} \mathrm{O}$-cooled QDs could be related to the surface states induced during the $\mathrm{H}_{2} \mathrm{O}$ cooling process compared to that of the QDs cooled in toluene or hexane (Figures 1(a) and 1(c)). The QD samples remixed with $\mathrm{H}_{2} \mathrm{O}$ after cooling first in toluene or hexane did not show any enhanced PL intensity. It is worth mentioning

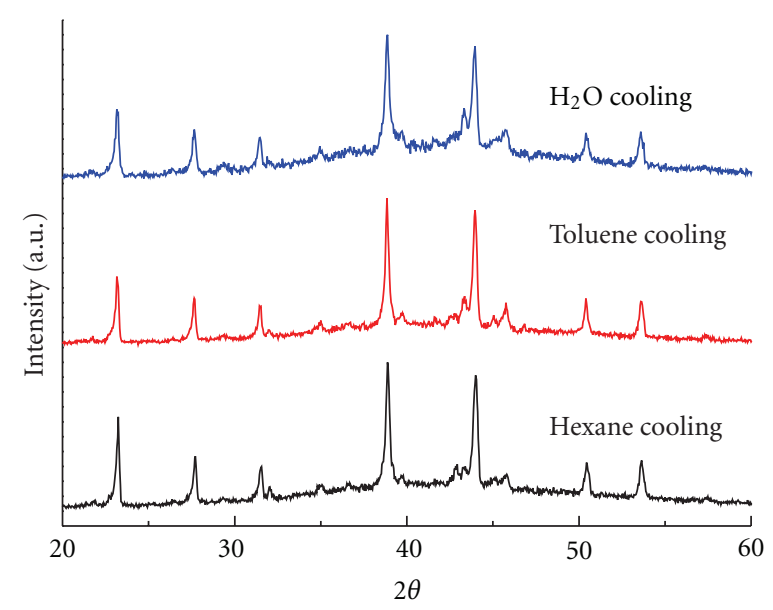

FIGURE 4: XRD patterns of CdSe QDs with different cooling solvents.

that no significant enhancement of PL was observed in watermixed QD samples after the cooling process using organic solvents. It means that PL enhanced mechanism related to $\mathrm{H}_{2} \mathrm{O}$-cooled QDs could be decided during the first cooling process after extracting the QDs from the reaction flask.

The PL intensity of the QDs can be further improved by low heat treatment (Figure 1(b)). The effects of heat treatment on the PL intensity of the QDs were investigated by changing the duration of the heat treatment at $120^{\circ} \mathrm{C}$. The QDs solutions were prepared by mixing the QDs and the different cooling solvents in vacuum oven under air conditions heated at $120^{\circ} \mathrm{C}$, the concentrations of the heat-treated QD solutions were also adjusted by diluting with toluene or hexane based on their absorption spectra as previously reported. After the heat treatment, the QD 

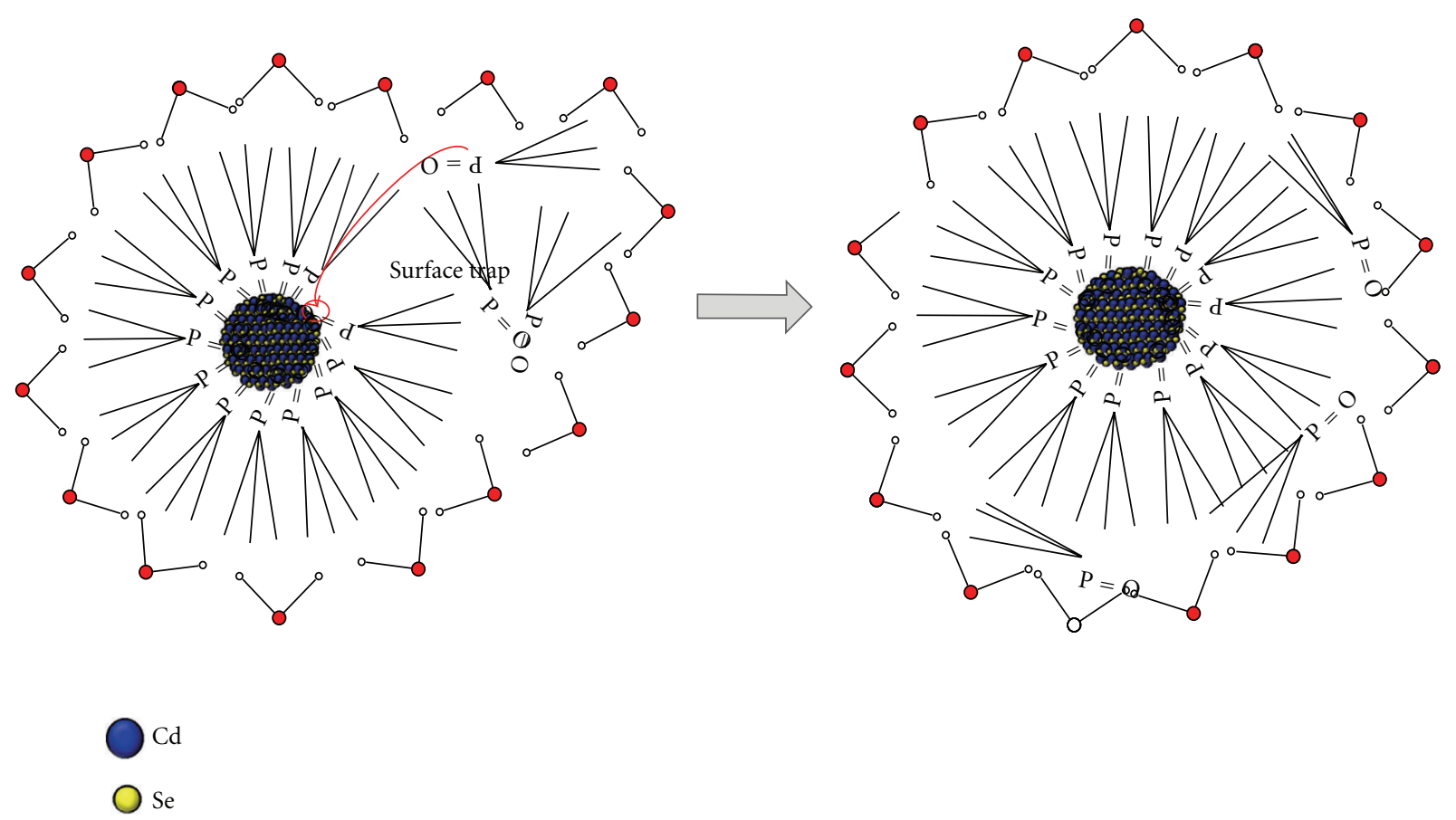

(a)

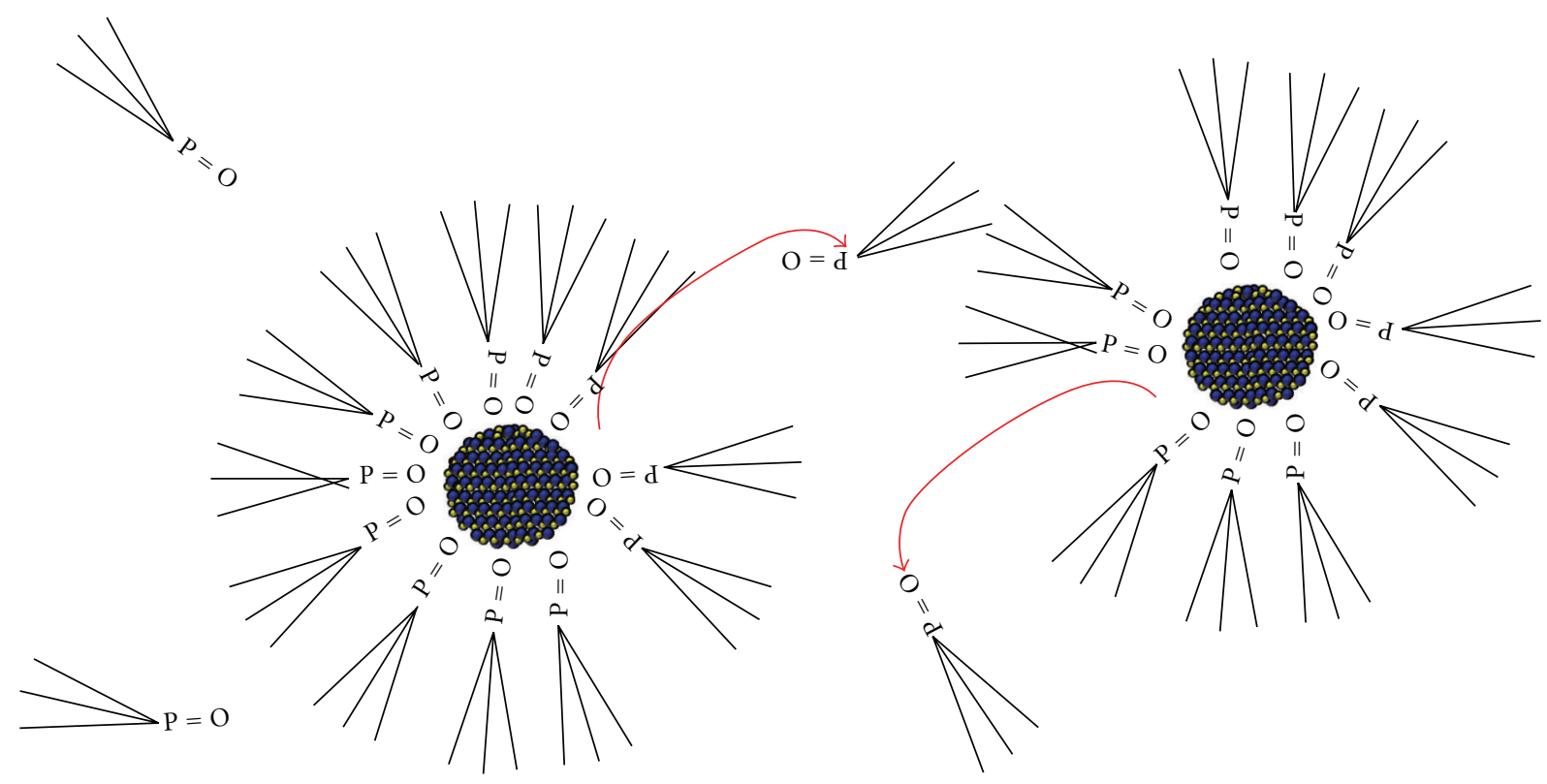

$\mathrm{Cd}$

(b)

FIGURE 5: Schematic of (a) the mechanism for the enhanced ligand passivation of QDs within $\mathrm{H}_{2} \mathrm{O}$ molecular containers, (b) the states of QDs and ligands within general organic solvents. 
solutions showed red shifts in the PL spectra which could be a result of additional growth during the heat treatment. The red-shifted values of each sample were different, which could be due to the difference in the effective heat energy contributing to the QDs growth related to the different vapor temperatures of the toluene and hexane. The high PL intensities of the CdSe QDs were shown to be at around three wavelengths of $525 \mathrm{~nm}, 542 \mathrm{~nm}$, and $552 \mathrm{~nm}$ during the extended heat treatment. Some periodic bright points were shown before the rapid deterioration of PL QY for the QDs (Figure 3).This result demonstrates a possible method to control the emission color of QDs related to bright point using postheat treatment. From the results of the periodic enhancement of PL QY, it is assumed that the optimized states of the QD surfaces showing the high PL QY could have existed during the postheat treatment. The as-synthesized core QDs with low PL QY could be due to the role of seeds with relatively more surface defects which could further increase the size of the seeds (showing the red-shift) accompanied by the effective recovery of surface defects with unreacted source compounds and ligands inside the $\mathrm{H}_{2} \mathrm{O}$ molecule containers during the postheat treatment. The reduction in PL QY of the QDs after passing the bright point could be attributed to the occurrence of perturbation in the optimized structure of the QDs at the bright point. The average PL QY at the bright point had values over approximately $80 \%$ and FWHM was approximately $22 \mathrm{~nm}$. The bright points with the high PL QY of the core QDs could not be confined to the orange color range but also existed in a wider color ranges during the heat treatment.

Cordero and coworkers demonstrated that PL increase by adsorbed water molecules [14]. Myung and coworkers had also shown PL increase by oxygen passivation of surface states [15]. In both cases, it was suggested that the PL enhancement of QDs is originated by the additional oxide layer formed on the QDs' surface. However, we suggest different mechanism for the PL enhancement because of two reasons. First, no significant differences in XRD data from all samples with different cooling process showed similar XRD patterns (Figure 4). The formation of oxide layer on the surface of water-cooled QDs could generate different XRD patterns/additional peaks related to oxide layer. Second, if the PL enhancement is originated by an oxide shell, a single optimal shell thickness showing the maximum PL intensity could exist. The PL intensity could be continuously decreased beyond the optimal shell thickness, which is generally observed during the shelling process [16]. However, the periodic bright points were shown during extended annealing of QDs within $\mathrm{H}_{2} \mathrm{O}$ molecule containers (Figure 3 ), which is significantly different behavior compared with the shelling process.

Therefore we suggested basic chemical mechanism for the dramatic improvement of QY in the $\mathrm{H}_{2} \mathrm{O}$ cooling process, and heat treatment is suggested in Figure 5(a). In the case of the aqueous synthesis, the CdTe QDs showed a very high PL QY at about $~ 90 \%$ QY [17] using the well-known Stöber method $[18,19]$. In contrast, general solvent synthesis methods avoid $\mathrm{H}_{2} \mathrm{O}$ and air which could induce oxidation and damage of the QDs. However, the luminescence properties of the QDs were not degraded when the as-synthesized QDs were mixed with $\mathrm{H}_{2} \mathrm{O}$. QDs with hydrophobic ligands have no chance for direct contact with $\mathrm{H}_{2} \mathrm{O}$ just like water and oil. This phenomenon can be applied to confine QDs within $\mathrm{H}_{2} \mathrm{O}$ molecule containers which could have a similar role as a reverse micelle. The distance between the confined QDs and the unreacted ligands/chemical compounds is much closer within the $\mathrm{H}_{2} \mathrm{O}$ molecule containers, which results in a decrease in the activation energy required for a chemical reaction. This environment within $\mathrm{H}_{2} \mathrm{O}$ molecule containers leads to a larger red-shift of $\mathrm{H}_{2} \mathrm{O}$-cooled QDs compared to solvent-cooled ones in the PL spectra with the same heattreatment time (Figure 1(b)). The high enhancement of PL QY can only be obtained from the $\mathrm{H}_{2} \mathrm{O}$-cooling process with the high-temperature QDs. Therefore, it is assumed that the confinement environment and temperature are necessary to enhance the PL QY of QDs by the efficient recovery of surface trap states during the $\mathrm{H}_{2} \mathrm{O}$ cooling process. In contrast, cooling using organic solvents could not meet both requirements and brought about the loss of surface ligands by generating a well-dispersive state (Figure $5(\mathrm{~b})$ ). Qu and Peng demonstrated that the PL bright point of QDs is in the orange color region which was observed in the QDs synthesis process [12]. However, our results show a more "bright point" and existed in more widely optical windows (Figure 2) due to the $\mathrm{H}_{2} \mathrm{O}$ cooling and postheat treatment process. These findings will significantly contribute to the development of bright core QDs and have also considerable implications for diverse application by further passivation processes accompanied with water such as silica coating, polymer encapsulation, and biofunctionalism. The suggested method provides an environmentally friendly method compared to cooling processes using organic solvents which generate $\mathrm{CO}_{2}$.

\section{Conclusions}

In conclusion, we have reproducibly demonstrated high enhancement of PL QY in the core of QDs and color controllability by finding additional bright points with an $\mathrm{H}_{2} \mathrm{O}$-cooling and postheat treatment process. It was experimentally confirmed that the $\mathrm{H}_{2} \mathrm{O}$ molecular containers could effectively passivate the surface defects of core QDs by the reaction of unreacted ligands/source compounds within $\mathrm{H}_{2} \mathrm{O}$ molecule containers. These findings can have significant potential implications in refuting the stereotypes that the $\mathrm{H}_{2} \mathrm{O}$ conceptually degrades the luminescent properties of QDs and has to be avoided in the synthesis and posttreatment of QDs. This suggested method also provides an environmentally friendly method without using organic solvents.

\section{Acknowledgment}

This paper was supported by the World Class University (WCU) project through the National Research Foundation (NRF) of Korea funded by the Ministry of Education, Science, and Technology (MEST; Grant no. R33-10070). 


\section{References}

[1] P. O. Anikeeva, J. E. Halpert, M. G. Bawendi, and V. Bulović, "Electroluminescence from a mixed red-green-blue colloidal quantum dot monolayer," Nano Letters, vol. 7, no. 8, pp. 21962200, 2007.

[2] H. Tada, H. Higuchi, T. M. Wanatabe, and N. Ohuchi, "In vivo real-time tracking of single quantum dots conjugated with monoclonal anti-HER2 antibody in tumors of mice," Cancer Research, vol. 67, no. 3, pp. 1138-1144, 2007.

[3] B. Farrow and P. V. Kamat, "CdSe quantum dot sensitized solar cells. Shuttling electrons through stacked carbon nanocups," Journal of the American Chemical Society, vol. 131, no. 31, pp. 11124-11131, 2009.

[4] M. G. Bawendi, P. J. Carroll, W. L. Wilson, and L. E. Brus, "Luminescence properties of CdSe quantum crystallites: resonance between interior and surface localized states," The Journal of Chemical Physics, vol. 96, no. 2, pp. 946-954, 1992.

[5] W. T. Al-Jamal, K. T. Al-Jamal, P. H. Bomans, P. M. Frederik, and K. Kostarelos, "Functionalized-quantum-dot-liposome hybrids as multimodal nanopartides for cancer," Small, vol. 4, no. 9, pp. 1406-1415, 2008.

[6] J. K. Kim, S. H. Lim, Y. Lee et al., "Conjugation of DNA to streptavidin-coated quantum dots for the real-time imaging of gene transfer into live cells," in Proceedings of the NSTI Nanotechnology Conference and Trade Show, vol. 3, pp. 379382, March 2004.

[7] X. Ji, J. Zheng, J. Xu et al., "(CdSe)ZnS quantum dots and organophosphorus hydrolase bioconjugate as biosensors for detection of paraoxon," Journal of Physical Chemistry B, vol. 109, no. 9, pp. 3793-3799, 2005.

[8] D. V. Talapin, I. Mekis, S. Götzinger, A. Kornowski, O. Benson, and $\mathrm{H}$. Weller, "CdSe/CdS/ZnS and CdSe/ZnSe/ZnS coreshell-shell nanocrystals," Journal of Physical Chemistry B, vol. 108, no. 49, pp. 18826-18831, 2004.

[9] D. Pan, Q. Wang, S. Jiang, X. Ji, and L. An, "Synthesis of extremely small CdSe and highly luminescent CdSe/CdS coreshell nanocrystals via a novel two-phase thermal approach," Advanced Materials, vol. 17, no. 2, pp. 176-179, 2005.

[10] G. W. Huang, C. Y. Chen, K. C. Wu, M. O. Ahmed, and P. T. Chou, "One-pot synthesis and characterization of highquality $\mathrm{CdSe} / \mathrm{ZnX}(\mathrm{X}=\mathrm{S}$, Se) nanocrystals via the $\mathrm{CdO}$ precursor," Journal of Crystal Growth, vol. 265, no. 1-2, pp. 250-259, 2004.

[11] H. Shen, H. Yuan, J. Z. Niu et al., "Phosphine-free synthesis of high-quality reverse type-I ZnSe/CdSe core with $\mathrm{CdS} / \mathrm{Cd}_{x} \mathrm{Zn}_{1-X} \mathrm{~S} / \mathrm{ZnS}$ multishell nanocrystals and their application for detection of human hepatitis B surface antigen," Nanotechnology, vol. 22, no. 37, Article ID 375602, 2011.

[12] L. Qu and X. Peng, "Control of photoluminescence properties of CdSe nanocrystals in growth," Journal of the American Chemical Society, vol. 124, no. 9, pp. 2049-2055, 2002.

[13] W. W. Yu, L. Qu, W. Guo, and X. Peng, "Experimental determination of the extinction coefficient of CdTe, CdSe, and CdS nanocrystals," Chemistry of Materials, vol. 15, no. 14, pp. 2854-2860, 2003.

[14] S. R. Cordero, P. J. Carson, R. A. Estabrook, G. F. Strouse, and S. K. Buratto, "Photo-activated luminescence of CdSe quantum dot monolayers," Journal of Physical Chemistry B, vol. 104, no. 51, pp. 12137-12142, 2000.

[15] N. Myung, Y. Bae, and A. J. Bard, "Enhancement of the photoluminescence of CdSe nanocrystals dispersed in $\mathrm{ChCl}_{3}$ by oxygen passivation of surface states," Nano Letters, vol. 3, no. 6, pp. 747-749, 2003.
[16] X. Zhong, R. Xie, Y. Zhang, T. Basché, and W. Knoll, "High-quality violet- To red-emitting $\mathrm{ZnSe} / \mathrm{CdSe}$ core/shell nanocrystals," Chemistry of Materials, vol. 17, no. 16, pp. 40384042, 2005.

[17] E. Ying, D. Li, S. Guo, S. Dong, and J. Wang, "Synthesis and bio-imaging application of highly luminescent mercaptosuccinic acid-coated CdTe nanocrystals," PLOS One, vol. 3, Article ID e2222, 2008.

[18] P. Yang, M. Ando, and N. Murase, "Encapsulation of emitting CdTe QDs within silica beads to retain initial photoluminescence efficiency," Journal of Colloid and Interface Science, vol. 316, no. 2, pp. 420-427, 2007.

[19] S. T. Selvan, T. T. Tan, and J. Y. Ying, "Robust, noncytotoxic, silica-coated CdSe quantum dots with efficient photoluminescence," Advanced Materials, vol. 17, no. 13, pp. 1620-1625, 2005. 

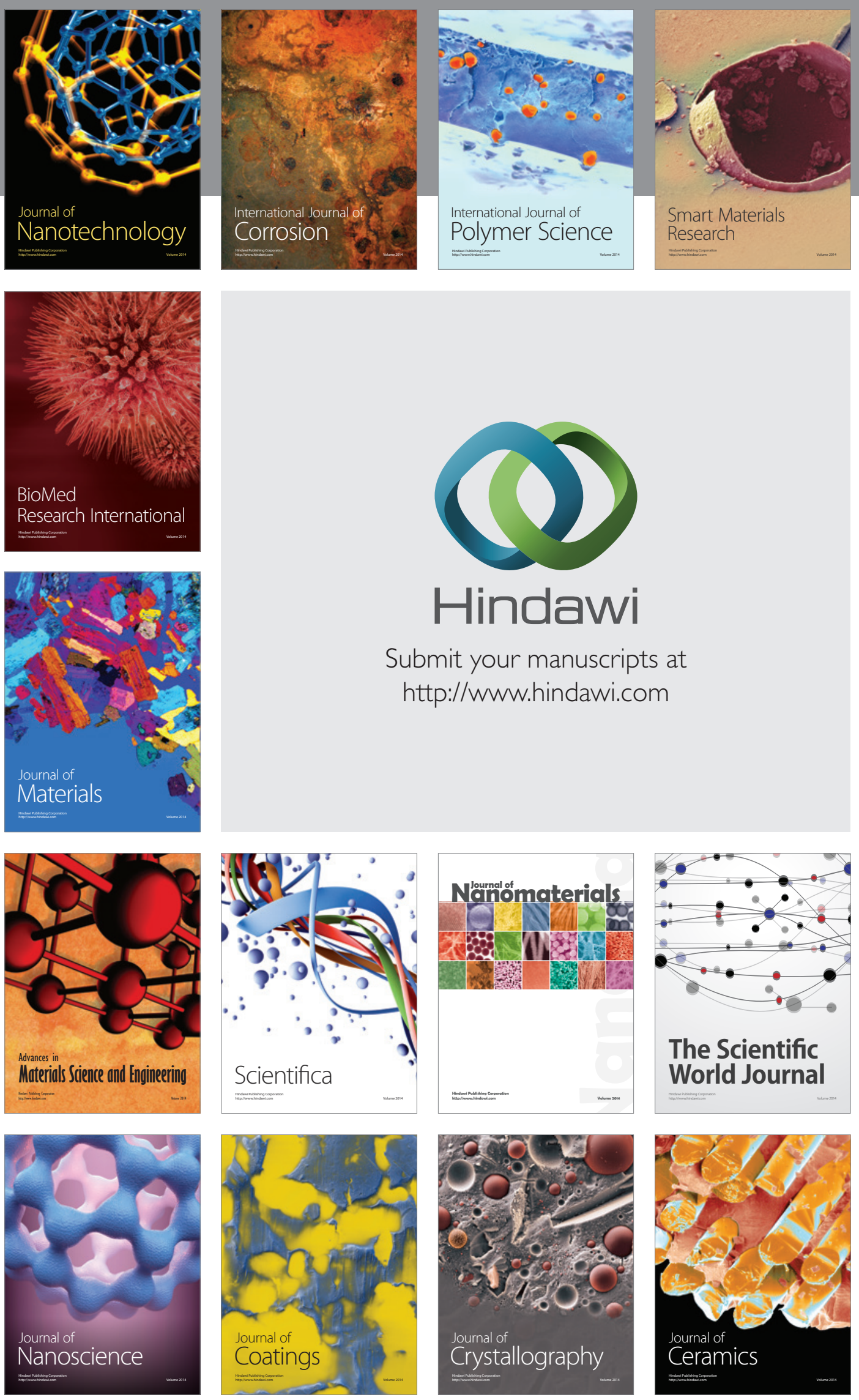

The Scientific World Journal

Submit your manuscripts at

http://www.hindawi.com

\section{World Journal}

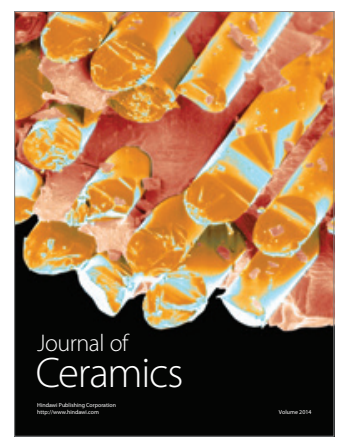

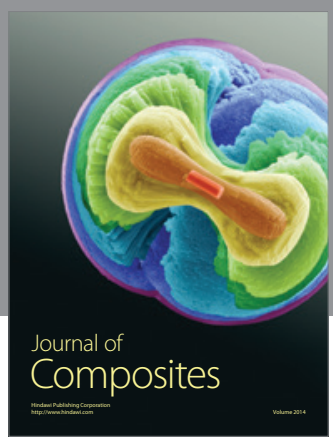
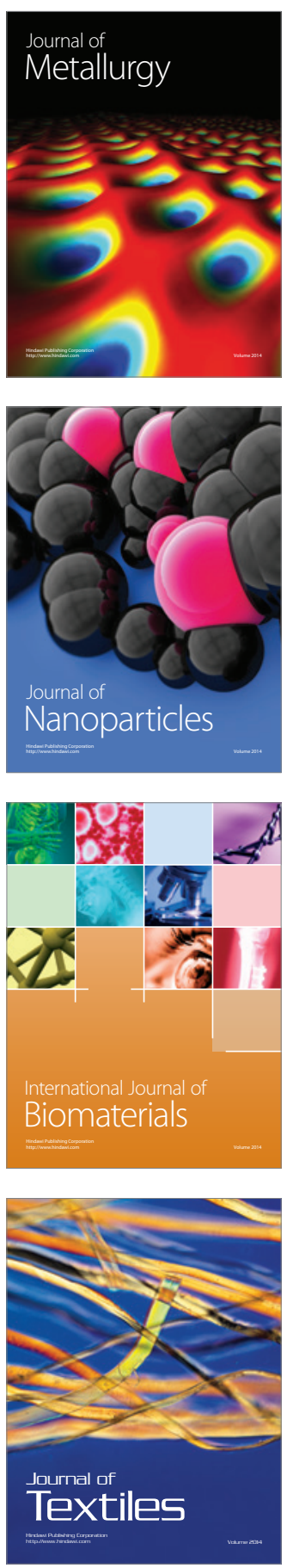